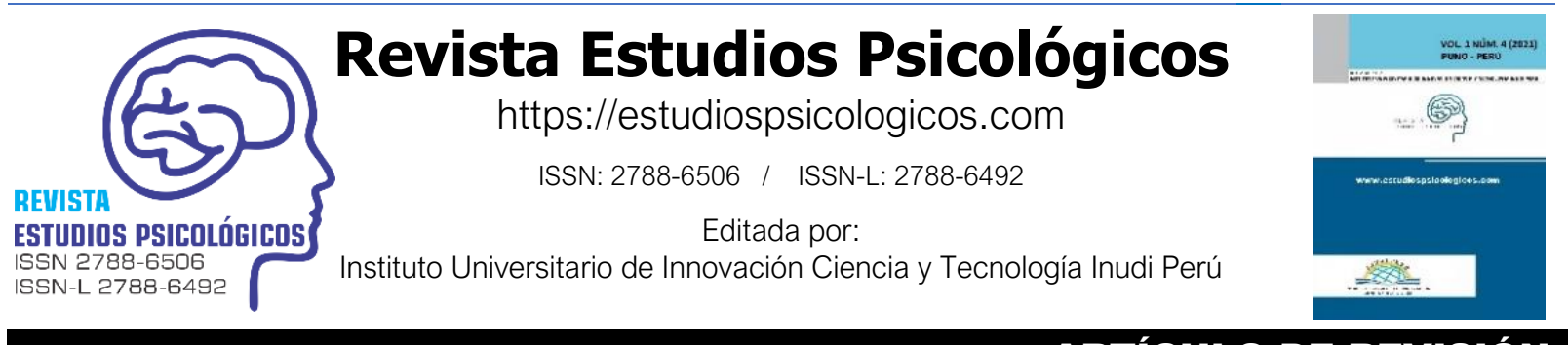

ARTÍCULO DE REVISIÓN

\title{
Apoyo emocional de la familia y éxito escolar en los estudiantes de educación básica
}

Family emotional support and school success in basic education students

Apoio emocional familiar e sucesso escolar em alunos do ensino fundamental

\author{
Edwin Sánchez ${ }^{1}$ \\ Universidad César Vallejo, Lima - Lima, Perú \\ (iD https://orcid.org/0000-0002-0892-5871 \\ Oscar Dávila \\ Universidad César Vallejo, Lima - Lima, Perú \\ (iD) https://orcid.org/0000-0001-6915-8373
}

DOI: https://doi.org/10.35622/j.rep.2022.01.001

Recibido 24/09/2020/ Aceptado 01/01/2022

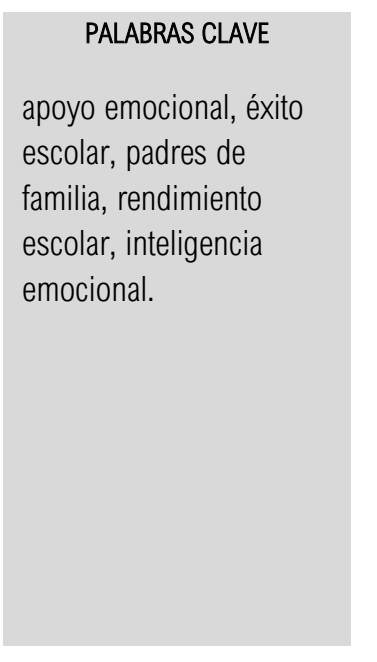

\section{KEYWORDS}

emotional support, school success, parents,
RESUMEN. El propósito de este estudio fue analizar el impacto del apoyo emocional en el éxito escolar mediante la revisión de publicaciones científicas iberoamericanas. El trabajo se enmarcó en el paradigma interpretativo, tuvo un enfoque cualitativo, con diseño documental y de alcance explicativo. Se revisaron artículos publicados entre los años 2017 y 2021, en revistas alojadas en bases de datos como Scopus, Ebsco, Web of Siencie, SciELO, Latindex y otras. Se pudo verificar que el acompañamiento emocional de los padres de familia impacta favorablemente en el éxito escolar de los niños y adolescentes. Además, entienden al apoyo que brindan a sus hijos como una tutoría parental, en algunos casos más constante que en otros. Durante la pandemia, ellos tuvieron la oportunidad de estar más cerca de sus hijos, compartieron y apoyaron en las actividades escolares. Los padres de familia forjan expectativas respecto al éxito escolar de sus hijos, pero su satisfacción de estas dependerá de la calidad de apoyo que les brinden. Estos resultados son importantes porque destacan la necesidad e importancia de que los padres de familia estén más presentes en la vida escolar de sus hijos si esperan que los estudiantes se comuniquen y relacionen asertivamente en distintos entornos, sean personas de bien, estudiantes exitosos y profesionales competitivos.

ABSTRACT. This study aimed to analyze the impact of emotional support on school success by reviewing Ibero-American scientific publications. The work was framed in the interpretive paradigm, had a qualitative approach, with documentary design and explanatory scope. Articles published between 2017 and 2021 were reviewed in journals hosted in databases such as Scopus, Ebsco, Web of Science, SciELO, Latindex, and others. It was found that the emotional accompaniment of

\footnotetext{
${ }^{1}$ Correspondencia: e.s.d@hotmail.com
} 


school performance,
emotional intelligence.

\section{PALAVRAS-CHAVE}

suporte emocional, sucesso escolar, pais, desempenho escolar, inteligência emocional. parents has a favorable impact on the school success of children and adolescents. In addition, they understand the support they provide to their children as parental tutoring, in some cases more constant than in others. During the pandemic, they had the opportunity to be closer to their children, shared and supported in school activities. Parents set expectations for their children's success in school, but their satisfaction with these will depend on the quality of support. These results are significant because they highlight the need and importance for parents to be more present in their children's school life if they expect students to communicate and interact assertively in different environments to be good people, successful students, and competitive professionals.

\begin{abstract}
RESUMO. 0 objetivo deste estudo foi analisar o impacto do suporte emocional no sucesso escolar por meio da revisão de publicações científicas ibero-americanas. 0 trabalho enquadrou-se no paradigma interpretativo, teve uma abordagem qualitativa, com desenho documental e âmbito explicativo. Os artigos publicados entre 2017 e 2021 foram revisados em periódicos hospedados em bases de dados como Scopus, Ebsco, Web of Siencie, SciELO, Latindex e outros. Verificou-se que o acompanhamento emocional dos pais impacta favoravelmente no sucesso escolar de crianças e adolescentes. Além disso, compreendem o apoio que prestam aos filhos na forma de tutoria parental, em alguns casos mais constante do que noutros. Durante a pandemia, eles tiveram a oportunidade de estar mais próximos dos filhos, compartilhados e apoiados nas atividades escolares. Os pais estabelecem expectativas para o sucesso de seus filhos na escola, mas sua satisfação dependerá da qualidade do apoio que fornecem. Esses resultados são importantes porque destacam a necessidade e a importância de os pais estarem mais presentes na vida escolar de seus filhos se esperam que os alunos se comuniquem e interajam de forma assertiva em diferentes ambientes, sejam boas pessoas, alunos bem-sucedidos e profissionais competitivos.
\end{abstract}

\title{
1. INTRODUCCIÓN
}

En el marco de la coyuntura social y de insalubridad mundial, surge la necesidad de revisar al impacto del apoyo emocional de los padres de familia en el rendimiento escolar y consecuente éxito de los estudiantes. Ahora más que nunca se hace hincapié en la necesidad de afecto, del apoyo emocional de padres a hijos. La situación de emergencia sanitaria acentuó la satisfacción de esta necesidad emocional en estudiantes, por la creciente inestabilidad emocional e incertidumbre producto de la pandemia y sus efectos (Castillo et al., 2021). Esta necesidad de apoyo emocional a los estudiantes se dio en todas las épocas y en diferentes circunstancias. Los investigadores continúan indagando sus implicancias en el aprendizaje. Por ejemplo, Lastre et al. (2017) analizaron la intervención paternal en la escolaridad, especialmente en niños de educación primaria, y establecieron que esta influye en el rendimiento escolar.

Durante la pandemia del Covid-19 surgieron una serie de dificultades y problemas poblacionales, laborales, sociales, económicos, las que modificaron diversas áreas de la actividad humana. El confinamiento, la consolidación del teletrabajo, la aparición de las sociedades digitales y la recesión económica cambiaron la rutina humana. "En el campo educativo concretamente los educandos fueron sometidos a una serie de vicisitudes en el aspecto emocional, en el proceso pedagógico se tuvo que ir sometiendo a otras formas de enseñanza" (Valdés, 2021, p. 20). Para los estudiantes no fue agradable el repentino cambio de modalidad educativa, que implicó la implementación de nuevas formas de enseñanza y aprendizaje (García, 2021). En tal 
contexto, el respaldo de la familia tiene que ver con el logro las competencias en las diferentes áreas curriculares. De ahí que las orientaciones, consejería y afecto que los padres brinden empoderamiento a los vástagos en la búsqueda del éxito escolar (Motha, 2018). Consecuentemente, la tutoría parental debe complementarse con la tutoría educativa brindada en la escuela, la calidad educativa requiere de una formación escolar y personal eficaz (Ferraces et al., 2020). La educación familiar, primera fase formativa de los educandos, permite que estos continúen con la formación escolar, antecedente de la educación superior (Tornés, 2021).

Los sistemas de respaldo paternal se constituyen en soportes relevantes en el proceso de formación de los educandos. Cabe destacar que la figura femenina es más influyente que la figura masculina. Aparentemente, la figura materna es más próxima, carismática, ofrece mayor respaldo a los hijos por el hecho de que la madre pasa más tiempo en casa (Lagos \& Luque, 2020). En palabras de Vaccotti (2019) los lazos de compromiso y afectividad de los progenitores y profesores son importantes en la formación escolar. De ellos depende los logros de aprendizaje de los niños y adolescentes. Sin la participación de los progenitores, los estudiantes tal vez no puedan superar las dificultades que se presenten en su vida escolar, porque tanto el papá como la mamá son fuentes de apoyo emocional.

Las emociones son señales que aportan información de lo que ocurre alrededor de una persona en relación con otras (Paris, 2020). Indican si la situación está bien o mal. El manejo y regulación de las emociones es propio de quien desarrolló su inteligencia emocional. Explica Goleman (2002) que esta es la capacidad del individuó para conocer los sentimientos suyos y de terceros y, a partir de ello, manejar sus relaciones interpersonales. Está comprobado que los individuos que manejan sus emociones se relacionan mejor con los demás. Tanto docentes como padres de familia usan su inteligencia emocional para relacionarse positivamente con los estudiantes, de suerte que la educación emocional se orienta a que los estudiantes sean emocionalmente saludables y equilibren sus capacidades cognitivas y emocionales (Viso, 2020). Esto tendrá importantes implicancias en su vida familiar, laboral y social. El desarrollo de las competencias emocionales es una tarea compartida entre los padres y los docentes, sirve para que el estudiante se desenvuelva asertivamente en cualquier entorno. La educación emocional es la vertebra el desarrollo personal de los individuos, responde a las necesidades emocionales y sociales de estos. Asimismo, permite prevenir la depresión, ansiedad, conflictos personales internos y externos (Rojas, 2019). En este sentido se puede afirmar que el individuo educado emocionalmente es capaz de conocer y entender sus emociones y las de los demás.

Los padres de familia tienen el deber de brindar apoyo emocional a sus hijos. Les inyectan las primeras dosis de amor, cariño y respeto. Les enseñan a valorarse a sí mismos, a respetar y tratar con afecto a los demás. Los padres balanceados forman hijos felices (Noguera, 2020). Un hogar, cuyos miembros son emocionalmente 
estables y maduros, es escenario ideal para el desarrollo integral de los niños, adolescentes y jóvenes, quienes aprenden del ejemplo que ofrecen sus padres y actúan como éstos fuera del hogar. El apoyo emocional de los padres de familia se convierte así en motor para el desarrollo saludable de los hijos, un punto de apoyo para lo que estos puedan lograr en su vida escolar o académica. Durante la pandemia del Covid-19, que obligó al confinamiento en el mundo entero, el teletrabajo y la teleeducación, fue ineludible el apoyo emocional de los padres de familia. Los hijos necesitaron más de sus padres, en especial los más pequeños en edad escolar. Como señala Rueda (2020) el apoyo moral o emocional de los padres es el deber de asistir a los hijos y ayudarlos a crecer sanos, a madurar, desarrollarse y formar su personalidad. Ese apoyo es inevitable en la edad escolar, ya que niños y adolescentes necesitan saber que sus padres son aliados para guiarlos en el complejo proceso de su aprendizaje. Papá y mamá estarán ahí siempre para echarles una mano cuando algo salga mal 0 para orientarlos cuando necesitan entender algo incomprensible en apariencia. Lamentablemente, esto no siempre es así. Hay personas que evaden sus responsabilidades de apoyo a los hijos, los dejan en segundo plano para divertirse, hacer vida social, pasar el tiempo frente al televisor o una pantalla de celular y, en el mejor de los casos, afrontar las cargas laborales o profesionales. Por eso es importante que los padres enseñen a sus hijos a ser independientes desde pequeños, digerir sus emociones y alcanzar el equilibrio (Sanz, 2020). De esta forma, los niños y adolescentes superarán las ausencias temporales de los padres, aprenderán a solucionar los problemas emergentes cuando estos no estén cerca.

El oportuno y adecuado apoyo emocional de padres de familia a los hijos evitará que estos caigan en desasosiego como resultado de un fracaso escolar, les dará fortaleza para sentirse capaces de superar lo imposible o comprender lo que al principio resulta incomprensible. Evitará que los estudiantes estén en riesgo de abandonar la escuela, les dará fortaleza para sobreponerse a fracasos iniciales. Existe vinculación entre el involucramiento de las familias en la educación de los hijos. Las que tienen mayor involucramiento en la vida escolar consiguen que los niños obtengan mejores resultados escolares (Razeto, 2016). Por esta razón, las autoridades escolares tienen que planificar más actividades para integrar las familias a la escuela y abordar temas de inteligencia emocional en reuniones con padres de familia.

La inteligencia emocional permite a los individuos gestionar sus emociones; entablar relaciones interpersonales armoniosas, gratas y satisfactorias. Su desarrollo en el hogar requiere de comunicación asertiva y permanente entre los integrantes de la familia. Caballero (2020) señala que la clave para construir buenas relaciones interpersonales se encuentra en el trato hacia los demás, la empatía, el control de las emociones y otros factores. Esa inteligencia emocional es modelada por los padres en el hogar, aprendida por los hijos y aplicada por estos en interacción con sus pares en la escuela y otros contextos. El apoyo emocional de los padres de familia a los hijos durante el proceso de aprendizaje involucra una elevada carga de inteligencia emocional para lograr que 
estos se sientan importantes, valorados y depositarios de elevadas expectativas personales, académicas y profesionales (futuras). Los niños, adolescentes y jóvenes que reciben y valoran el apoyo emocional de sus padres se sienten motivados y comprometidos con su rol de estudiantes, son perseverantes, se plantean metas y asumen los retos con decisión. Se esfuerzan para obtener excelentes resultados académicos, porque saben que sus padres atesoran justificadas expectativas por el éxito que puedan alcanzar.

Los padres participan (o deberían) en la educación de sus hijos. Consecuentemente, se plantean expectativas de éxito académico, esperan que sus hijos las satisfagan. Aspecto estudiado por Sánchez et al. (2016) quienes establecieron que la participación de los apoderados en la educación escolar se relaciona positivamente con el nivel educativo que logran los estudiantes. Los padres de familia responsables y comprometidos con la formación de sus hijos se plantean alturadas expectativas académicas (Razeto, 2016). La satisfacción de esta expectativa dependerá siempre de relaciones entre padres e hijos, así como del apoyo, material y emocional que estos reciban.

Se puede señalar con total seguridad que los aspectos socioemocionales juegan un papel importantísimo en la predisposición que deben mostrar los alumnos para realizar sus actividades escolares de manera eficiente y así conseguir el éxito escolar (Colomo et al., 2021). A través de este postulado se recomienda fortalecer apoyos estructurados a partir de programas alternativos, así como del accionar conjunto y colaborativo de todos los agentes educativos. De esta forma se potenciaría en los estudiantes la autoestima, la capacidad de amarse y valorarse. Se evitarían situaciones problemáticas y conflictos propios de educandos con baja o deficiente autoestima, nulo o escaso empoderamiento socioemocional (Vera et al., 2021).

Este estudio condujo a la revisión de publicaciones científicas que tienen como variables o categorías de estudio el apoyo emocional de los padres de familia y el éxito escolar. Es coherente y oportuno señalar que el soporte emocional favorece el bienestar psicológico y el desarrollo integral de los estudiantes. Por tal razón, resulta indispensable instar a los miembros de la comunidad educativa a la implementación de programas que comprometan el involucramiento de los padres de familia en la vida escolar de sus hijos, dado que de ello depende el éxito escolar.

Considerando lo expuesto, esta revisión tuvo como objetivo general: analizar el impacto del apoyo emocional en el éxito escolar mediante la revisión de publicaciones científicas iberoamericanas. De este se desprendieron tres objetivos específicos: (i) entender el significado que otorgan los padres al apoyo que brindan a sus hijos, (ii) caracterizar el apoyo emocional de los padres durante la pandemia y (iii) explicar las expectativas de los padres de familia respecto al éxito escolar de sus hijos. De esta forma se pretende consolidar aspectos teóricos 
que resulten de esta investigación, que sirvan para enfrentar los desafíos educativos del momento y el logro de la calidad educativa.

\section{MÉTODO}

El estudio se desarrolló dentro del paradigma interpretativo, sabiendo que éste se encarga de "analizar, entender e interpretar el accionar humano descrito en los artículos revisados y que tratan de dar significación a las relaciones de los individuos" (Rodríguez, 2013, p. 143). En el terreno educativo se trata de la comprensión e interpretación del accionar humano y sus implicancias en la vida social.

Se adoptó un enfoque cualitativo, porque se trató de alcanzar la comprensión de los fenómenos mediante una exploración de las perspectivas de los individuos vinculados a estos (R. Hernández \& Mendoza, 2018). Este estudio examinó la importancia del apoyo emocional de los padres de familia y el éxito escolar de los estudiantes, según la perspectiva de las publicaciones académicas realizadas entre los años 2017 y 2021. El tema se examina desde la coyuntura internacional con la finalidad de analizar su importancia en los sistemas educativos latinoamericanos.

Se siguió un diseño documental, ya que consultaron y revisaron artículos académicos publicados en los últimos cinco años, que dan cuenta del estudio de las categorías analizadas: apoyo emocional de los padres de familia y éxito escolar. Se recabó la información importante sobre las referidas categorías para analizarla e interpretarla (Arévalo et al., 2020; Arias, 2012).

Los resultados tienen un alcance explicativo. Se explicó la importancia del apoyo emocional de los padres de familia a sus hijos y las vinculaciones que este tiene con el éxito escolar (R. Hernández \& Mendoza, 2018). Los resultados servirán para que los educadores redirijan su atención sobre el apoyo emocional como factor decisivo para lograr que los estudiantes no se sientan solos durante el proceso de aprendizaje. Esto implica (por supuesto) que aquellos padres distantes de sus hijos a causa de las responsabilidades laborales, o a veces por preferir gastar su tiempo de ocio acudiendo a encuentros y reuniones con amigos, se acerquen más a estos y lo apoyen en el proceso hacia el éxito escolar.

La revisión incluyó una muestra de 28 artículos, correspondientes a distintos países iberoamericanos y publicados en diversas bases de datos (Scopus, Ebsco, Web Of Sience, SciELO, Lantidex y otras) caracterizadas por la rigurosidad científica de las publicaciones alojadas en ellas.

Como criterios de inclusión del material bibliográfico a revisar se consideró: que los artículos analizaran las categorías apoyo emocional (AE) de los padres de familia (PF) y éxito escolar (EE), las relaciones entre los padres de familia y la institución educativa, educación en tiempos de confinamiento, que los artículos se hubieran 
publicado entre los años 2017 y 2021. Obviamente, se excluyeron artículos con una temática distinta a la tratada en este estudio y que hubieran sido publicados en fecha anteriores a 2017.

Para la organización del material recolectado se utilizaron tablas comparativas y fichas analíticas que permitieron sistematizar los artículos con información clave de estos (Ñaupas et al., 2013). La técnica del análisis documental permitió examinar los artículos en busca de información relacionada con los objetivos planteados para orientar la revisión (Bisquerra, 2004).

El análisis incluyó la verificación del enfoque, diseño, las palabras clave, la revisión de los hallazgos principales de los investigadores, sus conclusiones, coincidencias y divergencias, así como vacíos encontrados.

\section{CONTENIDO}

La revisión del material bibliográfico permitió verificar los siguientes hallazgos.

\section{El apoyo emocional y el éxito escolar}

Los vínculos familiares tienen mucho valor para los estudiantes, favorecen la consecución de óptimos resultados educativos. Pérez et al. (2021) comprobaron en Cuba que, en los hogares cuyos padres se involucraron emocionalmente en la educación de sus hijos, estos obtuvieron mejores resultados académicos. Es así que compete a los padres de familia enseñar a sus hijos a asearse, protegerse frente a probables amenazas, saludar y mantener buenas relaciones con sus pares. Guzmán et al. (2019) comprobaron en Ecuador que los padres de familia tienen una poderosa influencia emocional en sus hijos; gestan los vínculos afectivos y sociales primigenios en la vida de sus vástagos y sientan las bases para relaciones interpersonales satisfactorias. La comunicación empática entre padres e hijos, la seguridad que les inculcan, los valores que practican y la motivación constante es la clave del éxito futuro de los niños y adolescentes.

En España, Solís y Aguiar (2017) comprobaron que los padres y madres de familia valoran su involucramiento en la vida escolar y de ello depende el éxito de sus hijos, esto es más notorio en el caso de los estudiantes que asisten a escuelas privadas. Sin embargo, la madre es quien está más cerca de ellos y les brinda mayor soporte emocional, el padre es un poco más distante, tal vez porque el trabajo y las responsabilidades profesionales se lo impide y también a medida que los hijos avanzan en el nivel educativo. Como señalan los españoles Cruz y Borjas (2019) es necesaria la colaboración de los padres en la adaptación de los hijos a la escuela y a la realización autónoma de los trabajos escolares; de ello depende el éxito escolar. Hay la necesidad de que desde las escuelas se diseñen y ejecuten planes de acción orientados al bienestar socioemocional de los estudiantes, se articulen esfuerzos de padres de familia y docentes. Así se evitaría el ausentismo, la repitencia y la deserción escolar (López et al., 2021). De igual manera, Ferraces et al. (2020) establecieron que el control y apoyo de los 
padres de familia a sus hijos tienen una influencia directa en el rendimiento, ello se complementa con el ambiente en que se desenvuelven los estudiantes, el grado de satisfacción que experimentan, los hábitos de estudio que hayan desarrollado y la autoeficacia.

En Argentina, C. Sánchez et al. (2019) Ilegaron a la conclusión de que se requiere desarrollar competencias socioemocionales en los estudiantes, de forma que estos sean conscientes de sus emociones, las gestionen con responsabilidad para convivir en armonía dentro y fuera de la escuela; pero esto exige la coordinación constante entre docentes y padres de familia.

Durante la pandemia, el acompañamiento de los padres de familia fue sumamente valioso. Valdés (2021) constató que el bienestar de los estudiantes va de la mano con la educación emocional y que su ausencia acarrea "trastornos emocionales (miedo, ansiedad, depresión) en docentes, padres de familia y alumnos" (p. 20). La educación emocional debería formar parte del currículo escolar por el fuerte impacto que tiene en la vida escolar de niños y adolescentes. La metamorfosis del sistema educativo presencial a la virtualización del mismo provocó una serie de vicisitudes emocionales en docentes, padres de familia y estudiantes, provocando inseguridad, inestabilidad y una serie de frustraciones qué superar.

Luego de la pandemia, la escuela no puede permanecer adormecida, es preciso explorar nuevos paradigmas educativos. En el hogar, los padres de familia (en especial las madres) brindarán soporte socioemocional a sus hijos preparándolos para superar las complicaciones provocadas por el confinamiento (Rubio \& Garduño, 2020). Todo tipo de alteración en las emociones del individuo requieren tratamiento urgente. Si se quiere tener estudiantes predispuestos a involucrarse en el proceso de aprendizaje-enseñanza, se les debe brindar un buen soporte socioemocional, motivarlos y garantizar su bienestar general desde el hogar. Aciertan Balsells et al. (2018) cuando afirman que las diversas afectaciones que trajo consigo la pandemia hizo ver cuán necesario es el apoyo emocional, que fortalece anímicamente a los estudiantes, agiliza su adecuación al ambiente de aprendizaje, les permite ejecutar con normalidad las actividades cotidianas y enfrentar felizmente los retos educativos.

Como afirma Fernández (2020) las emociones no solo son importantes en la salud y la vida, sino que la salud emocional es elemento prioritario en el aprendizaje y la enseñanza. Las situaciones estresantes que causan ansiedad en los estudiantes durante el confinamiento sirvieron para entender cuán importante es para estos aprender a manejar sus emociones. Más que nunca cobró importancia el soporte emocional brindado por los padres de familia, sin el cual los hijos no habrían logrado el equilibrio emocional necesario para sobrevivir al encierro. Definitivamente, el apalancamiento emocional que se ofrezca en los niveles educativos inferiores (tanto 
en la escuela como en el hogar) tiene incidencia en la formación de los estudiantes, fortalece su independencia, los vuelve sujetos seguros, estables y útiles a la sociedad, sienta las bases para una vida adulta eficaz y fructífera.

\section{Significado que otorgan los padres al apoyo emocional que brindan a sus hijos}

Los padres de familia requieren reconocer el valor del AE que brindan a sus hijos. Ya se vio que, los padres que apoyan a los estudiantes, son principalmente aquellos cuyos hijos estudian en escuelas privadas (más la madre que el padre). Muchos padres piensan que a los docentes corresponde ocuparse de la formación de sus hijos; no entienden que comparten esa responsabilidad con la escuela.

Flórez et al. (2017) señalan que los padres colombianos ven al acompañamiento como un trabajo colaborativo complementario a la labor de los docentes. El acompañamiento escolar no solo fortalece los vínculos entre padres e hijos, sino que los prepara para socializar e interactuar con el entorno y, por supuesto, para lograr aprendizajes de calidad. En México, C. Hernández et al. (2017) comprobaron que las madres de familia desempeñan una labora tutorial importante en casa, se involucran en la vida escolar de sus hijos, los motivan y fortalecen su autoestima, esto resulta determinante en los logros académicos. Efectivamente, hay necesidad de planificar intervenciones adecuadas para acrecentar el interés de los niños y adolescentes por la escuela. Otro factor cardinal es la comunicación entre padres e hijos y aumenta la confianza entre estos (Caballero, 2020). Además, los estudiantes comprenden la importancia de escuela en su desarrollo personal y preparación como futuros ciudadanos.

Hay perspectivas negativas respecto al significado que los padres de familia dan al acompañamiento emocional. Bellido (2021) comprobó en una falta de colaboración de los padres de familia en la vida escolar de sus hijos. Los padres son esclavos del panorama contemporáneo, sus recargadas labores y demás responsabilidades funcionan como excusas para descuidar la tutela formal de sus hijos, no se esfuerzan en cambiar esta realidad. Tal ausencia se convierte en un aspecto negativo con incidencia en las emociones de los estudiantes. En Perú, Madueño et al. (2020) verificaron que la relación de apoyo a los estudiantes tiene un matiz autoritario e impositivo (respecto a normas y límites). Esto no siempre genera un escenario favorable para el desarrollo socioemocional de los hijos. Identificaron también grupos de padres con actitudes positivas y menos autoritarias, estos más que imponer, se preocupan por convencer a sus hijos respecto a lo que es saludable o no para su desarrollo. Por su lado, Pineda y Chiappe (2018) afirman que los padres de familia necesitan estar empoderados para brindar un buen acompañamiento a sus hijos en el hogar; deben proteger a sus hijos, fortalecen su autoestima. No obstante, desde el hogar se debe promover la independencia y generar proyectos familiares de tipo productivo en que los estudiantes inviertan positivamente su tiempo. Así pues, el acompañamiento en el hogar es una oportunidad para valorar el rol formador de la familia. 
Los padres de familia entienden el apoyo socioemocional como un acompañamiento necesario para el desarrollo y formación integral de sus hijos. Ese apoyo es una suerte de tutoría familiar (complementaria a la de la escuela) con la que los se orientan a sus hijos, estrechan lazos afectivos y de comunicación con estos, los motivan, aumentan su confianza y autoestima, les ayudan a valorar a la escuela. Algunos están tan ausentes en la vida escolar de sus hijos que restan importancia a su rol como acompañantes. Otros son tan controladores e impositivos que asumen el apoyo como una oportunidad para ejercer su autoritarismo.

\section{El apoyo emocional durante la pandemia Covid-19}

El respaldo familiar a los estudiantes siempre ha sido motivo de estudio como factor influyente en el logro de aprendizajes esperados. Así, en su estudio, Guzmán et al. (2019) determinaron que el 68\% de padres interviene a veces en las actividades de apoyo a sus hijos y solo el $32 \%$ lo hacen de forma regular. Es preocupante, pues queda claro que la mayoría descuida el acompañamiento, una función tan importante en la formación integral de sus hijos. Aquel 68\% parece olvidar la influencia que ejercen en la vida emocional de sus hijos, dado que de ellos depende la gestión inicial de los vínculos afectivos de estos. También Corchuelo et al. (2019) comprobaron que el apoyo familiar (medido mediante el interés y participación de los padres en actividades de una institución educativa) influye en el interés y esfuerzo de los estudiantes. Por consiguiente, es preciso que se fortalezcan las conexiones entre escuela y padres de familia.

La crisis sanitaria, que dio lugar al confinamiento social, obligó a la búsqueda de nuevas estrategias de enseñanza. Una de ellas es el desarrollo de las clases mediante videoconferencias. Asimismo, puso a prueba el compromiso de los padres de familia para con el aprendizaje de sus hijos. Esto acentúo las obligaciones de la familia en cuanto a la educación de sus descendientes. De una u otra manera, las familias se involucraron más en la formación de sus hijos. Pérez et al. (2021) comprobaron que el 70,6\% de familias brindó apoyo emocional a sus hijos, igualmente, el 70,6\% los apoyó en teleclases. Esto demuestra que obtuvieron mejores resultados académicos quienes recibieron más apoyo emocional de sus progenitores. Se abordó la colaboración de la familia en las tareas escolares en época de pandemia, Muñoz y Lluch (2020) manifiestan que la colaboración familiar contribuye a la mejora de los aprendizajes. Pero debe prestarse atención a ciertos riesgos en los que a veces no se repara: exceso de tareas escolares, generación de confusión en los estudiantes y padres de familia, ansiedad sobreviniente, miedo e incertidumbre. Esto se evitaría con una previa y razonable planificación que estime las dificultades emergentes a los estudiantes y sus familiares ante la ausencia de un maestro, cuyo rol en la educación presencia difiere ostensiblemente al de la enseñanza remota. Los riesgos se agravan cuando se trata de familias vulnerables cuya situación paupérrima debilita sus posibilidades de acceder a una conexión 0 los miembros familiares carecen de preparación para ayudar con las tareas escolares a sus hijos. Por ejemplo, 
Bravo y Oviedo (2020) comprobaron la relación entre el aislamiento social motivado por la pandemia y el estrés emocional de padres de familia vulnerables, obviamente, ocurre lo mismo con los estudiantes. La salud emocional debe ser un centro de atención durante la planificación de los aprendizajes. Es necesario dosificar las tareas, teniendo en cuenta que muchas responsabilidades de los docentes son trasladadas a los padres de familia.

\section{Expectativas de los padres respecto al éxito escolar de sus hijos}

La educación virtual durante la pandemia supuso una serie de desafíos: el compromiso de los estudiantes, la autonomía y automotivación; el autocontrol, la autodeterminación y autodisciplina. Gutiérrez y Espinel (2021) para cumplir con los retos que plantearan los docentes. Del lado de los padres de familia, el compromiso y apoyo decidido a sus hijos, si pretendían que estos satisficieran sus expectativas. Una educación virtualizada será siempre una oportunidad más que una improvisación impuesta. El éxito en ella depende de las estrategias didácticas empleadas por los docentes, la responsabilidad plena de los estudiantes, el apoyo y supervisión amigable de los padres de familia. No obstante, como señalan Expósito y Marsollier (2020) se debe tener en cuenta que la generalización de la educación virtual en pandemia develó las enormes desigualdades socioeducativas con implicancias preocupantes en el proceso de aprendizaje. Los estudiantes de regiones menos favorecidas y familias con grietas de vulnerabilidad más pronunciadas tuvieron más dificultades para cumplir con sus responsabilidades escolares. Y fueron sus padres quienes tal vez experimentaron más frustraciones ante las imposibilidades que sus hijos no pudieron superar o que complicaron su labor escolar.

Las expectativas de los padres de familia deben ir de la mano con su mediación, brindando a sus hijos el apoyo emocional (Halpern et al., 2021). Este apoyo responsable incide en los resultados de aprendizaje, es la mejor forma de expresar las expectativas por él éxito académico de los hijos. Aquellos padres de familia que no se involucran en forma efectiva en el proceso académico de sus hijos no pueden plantearse grandes expectativas, solo esperar la cosecha de sus descuidos y abandonos. Aquí cobra relevancia el estudio de Tua (2021) quien comprobó en Venezuela que durante el confinamiento hay una tendencia mayoritaria de padres de familia que no se involucran positivamente en el quehacer educativo de sus hijos. Además, ellos no establecen normas dentro del hogar para regular el comportamiento académico de los hijos e instaurar un equilibrio entre afecto y autoridad (Sanz, 2020). Como consecuencia de ello, los estudiantes carecen de hábitos de estudio o estos son deficientes. Por consiguiente, estos padres de familia no pueden plantearse grandes expectativas académicas para sus hijos; estos últimos corren el riesgo de fracasar y, en otros casos, desertar de la escuela.

La deserción escolar fue una consecuencia inevitable en los lugares donde los estudiantes enfrentaban una serie de dificultades: ausencia de conectividad, lentitud en la red, falta de herramientas tecnológicas, escasos 
recursos que impedían adquirir un dispositivo (tableta, PC, teléfono celular), dificultad de los sus padres para apoyarlos (en el caso de los niños de inicial y primeros grados de educación primaria), entre otros. Indudablemente, en contextos familiares difíciles, la deserción trajo por los suelos las expectativas de los padres acerca del éxito escolar de sus hijos. Sin embargo, Pacha y Rodríguez (2021) destacan que las autoridades educativas tuvieron reacciones inmediatas para favorecer a los grupos vulnerables. También resaltan la disposición de la mayoría de estudiantes a responder al plan educativo implementado por el gobierno. De cualquier forma, el apoyo de los padres de familia es un factor clave para que los hijos alcancen el éxito escolar en condiciones adversas. Las expectativas que aquellos puedan plantearse respecto al éxito escolar de sus hijos, que dependerá del apoyo que les brinden. Estas expectativas dependen además del desempeño de los docentes. Empero, Villanueva et al. (2021) comprobaron, por ejemplo, en Perú, que en tiempos de pandemia los estudiantes y padres de familia consideraban como excelente el desempeño de los docentes; aunque un mínimo porcentaje calificaba tal desempeño como regular y deficiente.

La familia juega un papel fundamental en el proceso educativo de los niños y adolescentes. Alonso et al. (2021) indican que la pandemia Covid causó grandes cambios en la dinámica familiar. La educación de los niños, adolescentes y jóvenes resultó afectada por esas metamorfosis. Para afrontar los retos pandémicos, los padres de familia debieron prodigar amor a sus hijos, acompañarlos, comprometerse con la ayuda que estos necesitaban. La motivación constante y la promoción de autonomía beneficiaba la continuidad de los estudios y él éxito escolar. Las emociones son relevantes e importantes en el desarrollo humano y por supuesto, en la educación de este (Buitrago \& Molina, 2021). No basta el apoyo emocional que se brinda en el hogar, también es necesario que los docentes brinden asistencia emocional a los estudiantes en la escuela. Los cambios experimentados durante la pandemia Covid-19 exigieron que docentes y padres de familia brindasen apoyo emocional a los estudiantes; más si esperaban que sus expectativas respecto al logro de aprendizajes en sus vástagos se vieran satisfechas.

Los padres esperan que sus hijos sean dedicados y responsables con sus deberes escolares, que obtengan los mejores resultados académicos, superen los diferentes niveles educativos y se conviertan así en personas de bien, ciudadanos íntegros y profesionales exitosos. Pero esto se quedará en simples anhelos o expectativas si, como progenitores responsables y preocupados por el bienestar de sus vástagos no les proveen los recursos materiales para desenvolverse sin dificultades ni carencias en la escuela, los acompañan con palabras de aliento y apoyan cada vez que los necesitan para realizar una tarea escolar complicada. De este modo los estudiantes verán que no están solos; tienen en el hogar a sus padres, dispuestos a ayudarles, y en la escuela a sus profesores, que les brindan asistencia académica especializada. 


\section{DISCUSIÓN}

El apoyo emocional que brindan los padres de familia a los estudiantes favorece el éxito escolar (Razeto, 2016; A. Sánchez et al., 2016). En tiempos de normalidad, ese apoyo se evidencia en el involucramiento de los progenitores en las actividades escolares de sus hijos, en su presencia frecuente en la institución educativa y en las conversaciones agendadas con los docentes para indagar sobre los logros de aprendizaje de los discentes. Durante la pandemia, este apoyo fue muy necesario e inevitable, los estudiantes tenían dificultades para entender muchos aspectos relacionados con los trabajos escolares y ningún contacto directo con los docentes para preguntarles. En efecto, los hallazgos de Ferraces et al. (2020) indican que el "apoyo y control" de los padres favorece el rendimiento de los estudiantes; y que concurren con estos otros factores coadyuvantes (ambiente, satisfacción que experimenten, sus hábitos de estudio y la autoeficacia). En el mismo sentido están las comprobaciones de Pérez et al. (2021), Rubio y Garduño (2020) y Guzmán et al. (2019) quienes obtuvieron evidencia para afirmar que el involucramiento de los padres de familia en las actividades y tareas escolares de sus hijos tiene un reflejo favorable en los resultados de aprendizaje. Por supuesto, el impacto es mayor en la educación básica. Pero debe considerarse que no basta el soporte emocional brindado por los padres de familia, el éxito escolar requiere movilizar una serie de factores materiales (recursos de aprendizaje), afectivos (acompañamiento, motivación) y contextuales (ambiente agradable, buenas relaciones interpersonales) que impactan en el logro de los aprendizajes. También la interacción sólida y dinámica entre docentes, estudiantes y padres de familia agiliza el proceso de aprendizaje en los estudiantes.

Se trata de evitar el ausentismo, la deserción y el fracaso escolar que, lamentablemente, merodearon durante la pandemia. Como coincidieron en señalar Balsells et al. (2018); Fernández (2020) y Valdés (2021) muchos estudiantes experimentaron fracasos y frustraciones ante las dificultades para comprender una tarea y por efecto del mismo confinamiento. En ese panorama fue más que necesario el sostén emocional de los padres de familia. Sin duda, como señalan C. Sánchez et al. (2019) en una estrategia conjunta entre docentes y padres de familia, es preciso desarrollar competencias socioemocionales en los estudiantes, de modo que estos sepan gestionar responsablemente sus emociones y convivir en forma armónica en contextos familiares (padres de familia y estudiantes), escolares (estudiantes y docentes) y profesionales (docentes). Abunda evidencia de que el apoyo emocional impacta en el aprendizaje, determina los resultados educativos y, por ende, el éxito escolar. Entonces, lo que los padres puedan hacer por sus hijos los predispone favorablemente al compromiso con sus responsabilidades escolares. En vista de ello, docentes y padres de familia necesitan estrechar el contacto emocional con sus hijos y coordinar con los docentes actividades y acciones educativas en beneficio del bienestar personal y académico de estos. La educación emocional es cada vez más necesaria para hablar de 
una verdadera formación integral (Rojas, 2019; Viso, 2020). Los padres con una inteligencia emocional bien desarrollada formarán hijos seguros, estables, emocionalmente felices (Noguera, 2020; Paris, 2020).

Entre los aspectos desfavorables advertidos en la revisión está el hecho de que los padres de familia son quienes menos se involucran en el quehacer escolar de sus hijos (las mamás están más cerca de estos). Faltan programas y planes de acción para involucrar emocionalmente a los padres en la vida escolar de niños y adolescentes. Además, los docentes tienen el reto de idear estrategias para acercar a los padres de familia a la escuela y comprometerlos en el aprendizaje de sus hijos.

Pero ¿qué significa para los padres el apoyo emocional que brindan a sus hijos? La mayor parte de padres y madres entienden que el apoyo emocional a sus hijos es un acompañamiento necesario para el desarrollo integral de estos y se complementa con la tutoría de los maestros en la escuela. Por esta razón, se debe incidir en la sensibilización a las familias, para que estas entiendan la seriedad e importancia del apoyo emocional que brindan a sus hijos, especialmente porque algunos descuidan este aspecto porque desconocen su impacto en los aprendizajes esperados y el éxito escolar. La mayoría de padres de familia entiende que la proximidad y apoyo a sus hijos no solo fortalece los lazos parentales, sino que es un estímulo para las interacciones sociales de niños y adolescentes en la escuela y comunidad (Flórez et al., 2017). Esto parecen entenderlo muy bien las madres, quizá porque en la mayoría de casos son quienes pasan más tiempo cerca de los hijos, enterándose de sus experiencias, necesidades urgentes, fracasos y éxitos, ellas saben suplir el vacío dejado por los padres, pero no tiene que ser así (C. Hernández et al., 2017). Los estudiantes necesitan saber que papá y mamá están pendientes de ellos, se interesan por lo que les pasa, que cuentan con su apoyo constante para superar las dificultades que surjan en la escuela.

En algunos casos, la paupérrima intervención de los padres y el poco significado que dan al apoyo emocional es producto de lo que ellos vivieron en su niñez. Es preciso pues que los papás logren un equilibrio entre sus responsabilidades laborales o profesionales (Bellido, 2021) y dediquen más tiempo al acompañamiento de sus hijos; su ausencia en la vida escolar de estos produce vacíos y menoscabos emocionales que afectan el normal desarrollo de aquellos. Como hacen notar Madueño et al. (2020) se debe fortalecer la presencia de la figura paterna y que esta no sea autoritaria e impositiva. Los niños y adolescentes necesitan que papá y mamá los orienten, aconsejen y acompañen en el complejo proceso de su aprendizaje escolar. Es una minoría la que realmente toma conciencia y acciona en forma responsable. Pineda y Chiappe (2018) no se equivocan cuando afirman que los padres (en general) deben ser empoderados para que brinden el apoyo emocional que sus hijos necesitan para superar con éxito la etapa escolar y prosperen hacia la superior. 
Durante la pandemia Covid-19, el apoyo emocional de los padres de familia fue un factor determinante. Con el confinamiento se enfrentó algunos retos y dificultades. Algunos esquemas de vida y algunos modos de llevar a cabo ciertos servicios se vieron modificados; la formación de los hijos no escapa a estas variaciones. La pandemia Covid-19 transformó la forma de vivir de la humanidad. El confinamiento como medida preventiva demandó mayor respaldo emocional de la familia a los estudiantes, por la inseguridad, incertidumbre, caos y resquebrajamiento emocional en toda la población; más todavía en los menores (Muñoz y Lluch, 2020). Aunque debe recalcarse que el apoyo familiar a los hijos debe darse independientemente de cualquier suceso 0 acontecimiento particular, en vista de los resultados favorables en el plano educativo. Lo bueno de la pandemia es que permitió el acercamiento familiar, estrechamiento de vínculos de amor y afecto, mejores relaciones interpersonales en el seno familiar, y más apoyo familiar en labores académicas, lo que debía reflejarse en los resultados educativos y el éxito escolar. Los padres de familia brindaron apoyo emocional y académico a sus hijos durante las clases virtuales (Pérez et al., 2021). Sin embargo, téngase en cuenta que no todo brilló como el sol, la situación se tornó complicada en el caso de las familias vulnerables, que tienen una situación económica difícil, viven en condiciones de pobreza, en zonas aisladas y sin posibilidades de conexión a una red. En el peor de los casos, no cuentan con herramientas tecnológicas para que sus hijos asistan a las clases virtuales. Precisamente, Bravo y Oviedo (2020) encontraron relación entre el aislamiento social obligado por la pandemia y el estrés emocional de padres y madres pertenecientes a familias vulnerables. El ejemplo sirve como evidencia de las brechas económicas, sociales culturales y tecnológicas que subsisten en la realidad iberoamericana y sobre las que los desposeídos todavía no pueden dar un salto cualitativo. En las próximas décadas, la mirada de los gobiernos debe apuntar a ese horizonte turbio de la realidad para gestionar los recursos del estado en beneficio de quienes más necesitan.

La presencia emotiva de mamá o papá en la vida académica de un niño 0 adolescente estimula el entusiasmo de estos, los inspira a querer ser mejores cada día. En la medida en que los padres y madres se interesen por lo que hacen sus hijos y participen activamente en las diferentes actividades propuestas por la institución educativa, lograrán que estos se interesen por aprender y valoren la escuela (Corchuelo et al., 2019). Aquí hay que mencionar a Guzmán et al. (2019), quien comprobó lamentablemente que la mayoría de padres se involucran ocasionalmente en la vida escolar de sus hijos, y solo el 32\% se involucra regularmente. Esto ocurre en todos los niveles y clase sociales, en unos más que en otros. Los ricos, porque andan muy ocupados en sus negocios y relaciones sociales y, pese al nivel cultural o académico que poseen, no apoyan a sus hijos con las tarreas escolares. Los pobres, porque deben trabajar muchas horas para ganar lo necesario o porque no tienen la preparación necesaria para brindarles soporte académico a sus hijos, en algunos casos también porque no les interesa, prefieren estar con los amigos o frente al televisor. Es necesaria la buena voluntad de los padres 
de familia para que con su apoyo los estudiantes tengan cierto éxito escolar. Pero la buena voluntad de aquellos no basta para que los niños (en especial los que pertenecen a sectores de pobreza) aprendan mejor, sino que debe ir de la mano con mejores condiciones de vida resultantes de políticas sociales que priorizan las necesidades de quienes menos tienen.

Las expectativas que los padres de familia tienen frente al éxito escolar de sus hijos es otro tema complejo en la educación virtual. Para que las expectativas académicas tengan validez y los estudiantes encuentren el interés y motivación para esforzarse en las actividades de aprendizaje. Se reclama, en primer lugar: el apoyo emocional de los padres de familia en el hogar y en segundo lugar de los docentes en la escuela (Alonso et al., 2021; Buitrago \& Molina, 2021; Rueda, 2020). Así los estudiantes tendrán la inspiración y deseos de dar lo mejor de sí para superar los retos académicos. Pero si los padres de familia no brindan soporte emocional y material a sus hijos, no pueden exigir ni esperar demasiado de estos. Si demandan más de lo que estos pueden dar considerando las difíciles condiciones en que asisten a las clases virtuales, solo pueden conseguir que estos detesten la escuela, reaccionen en forma negativa o caigan en estados de estrés y depresión profundos. El apoyo emocional que brindan tiene un fuerte impacto en el aprendizaje de los hijos (Alonso et al., 2021; Halpern et al., 2021). Ese apoyo involucra también proveerles materiales de estudio, recursos tecnológicos, soporte en el manejo de Tecnologías de Información y Comunicación (TIC). En el caso de los estudiantes más pequeños). Y si el compromiso, el apoyo y motivación faltan, los estudiantes no tienen muchas posibilidades de éxito escolar. Entonces, los padres de familia no pueden plantearse muchas expectativas. Al respecto, en Venezuela, Tua (2021) verificó que muchos padres de familia no asumen su rol, evaden su responsabilidad parental y no se involucran en el proceso de aprendizaje de sus hijos. Por fuerza de la gravedad, esto devendrá en un lamentable fracaso escolar, que solo puede evitarse con la oportuna reacción de los padres. Así como la asistencia emocional y material que brinden a sus hijos.

La deserción escolar se acrecentó, en tiempos de pandemia fue el fantasma que recorrió el país entero (Pachay \& Rodríguez, 2021). Los estudiantes afectados por las brechas socioeducativas, que no disponían de herramientas tecnológicas para conectarse a sus clases virtuales. Algunos vivían en zona alejadas a donde no llegaba la señal de internet, televisión o radio o sentían que no comprendían lo explicado por los docentes desde la distancia, desertaron. Prefirieron esperar que la pandemia terminase y se produjera el regreso a las aulas. Para evitarlo era muy importante lo que pudieran hacer los docentes (estratégica y metodológicamente), los propios estudiantes (deseo de vencer las dificultades, automotivación) y los padres de familia (apoyo absoluto a sus hijos, búsqueda de recursos que los ayudasen a culminar el año escolar). Por ejemplo, resulta importante destacar el estudio de Villanueva et al. (2021) quienes verificaron que la mayoría de estudiantes y 
padres de familia percibían como excelente el desempeño de los docentes durante la pandemia y un mínimo porcentaje de estos lo tildaban de regular o deficiente.

\section{CONCLUSIONES}

El apoyo emocional que brindan los padres de familia impacta favorablemente en el éxito escolar. Las muestras de afecto constante, la motivación, la asistencia en las tareas escolares difíciles, las palabras de aliento, el suministro de materiales de estudio y herramientas TIC necesarias en el hogar, la asistencia a las reuniones en la institución educativa, la preocupación por saber en diálogo con los docentes cómo avanzan sus hijos en el proceso educativo son claves en la formación integral de los niños y adolescentes. La inteligencia emocional permite a los padres de familia reconocer sus emociones y las de sus hijos, atender las necesidades de apoyo de estos y acompañarlos positivamente en su vida escolar. Esa misma inteligencia emocional es aprendida por los hijos y utilizada en sus interacciones dentro del hogar, en la escuela y comunidad. Esto permite comunicarse asertivamente, entablar relaciones interpersonales sólidas, participar activamente en las actividades de aprendizaje y alcanzar el éxito escolar.

Para los padres de familia, el apoyo que brindan a sus hijos debe ser una oportunidad para estar más presentes en la vida de estos, en su actividad escolar, en sus éxitos y fracasos, en sus dificultades y en los momentos de alegría y satisfacción. Se trata de una tutoría parental desde el hogar. Sin embargo, no todos los padres asumen responsablemente ese deber. Hay padres de familia que están ausentes en la vida escolar de sus hijos debido a las responsabilidades laborales o profesionales, la vida social o actividades de diversión con amigos y conocidos. Inclusive, dentro del hogar, la televisión y actualmente los dispositivos celulares se convierten en enemigos de la unidad familiar.

Durante el confinamiento obligado por la pandemia del Covid-19, los padres de familia permanecieron más tiempo en el hogar, la familia pasó más tiempo junta; sin embargo, no todos aprovecharon esa oportunidad para apoyar a sus hijos en los quehaceres de la escuela. Tal vez unos lo hicieron más que otros, los más preparados pudieron brindar asistencia con las tareas escolares y asegurar así el éxito escolar en el complejo panorama de la pandemia que obligó a una educación virtual. Pero hubo también grupo de padres cuyo nivel educativo no les permitió dar a sus hijos el apoyo que requería el desarrollo de algunas actividades de aprendizaje. Aun así, la mayoría de padres de familia se esforzaron por brindar soporte emocional a sus hijos. Y claro que también hubo deserción escolar en aquellos sectores de la población donde los padres no pudieron asistir a sus hijos durante las clases virtuales. La dificultad para acceder a una herramienta tecnológica con capacidad suficiente para conectarse a las plataformas educativas, el no contar con señal de internet o la conectividad lenta e inestable. En estos casos, el apoyo parental fue de la mano con la frustración y el fracaso escolar. En tiempos 
de confinamiento, el éxito escolar es un reto e involucra necesariamente a diversos estamentos gubernamentales y no gubernamentales. La familia juega un papel preponderante, su involucramiento emocional y psicológico incide favorablemente en la vida escolar de los niños y adolescentes, debilita los efectos de la inestabilidad, inseguridad e incertidumbre.

Todo padre de familia se forma expectativas respecto al éxito escolar de sus hijos. Pero para que suceda como lo esperan, no basta con vestir a los vástagos con un uniforme, comprarles los útiles escolares y enviarlos a la escuela. Es preciso que los niños y adolescentes sientan que cuentan con el apoyo permanente de papá y mamá, que estos están a su lado para ayudarles en los momentos más difíciles y con aquellas tareas escolares complicadas. Los estudiantes necesitan saber que sus padres esperan mucho de ellos, que desean verlos convertidos en personas íntegras, ciudadanos responsables y profesionales exitosos. Teniendo esto como horizonte ideal, los acompañan con palabras de amor y afecto, los apoyan con responsabilidad, constancia y deber parental. En suma, lo que los hijos puedan lograr o no en su vida académica depende mucho del apoyo material y emocional que los padres les brinden en cada momento de la vida escolar.

\section{Conflicto de intereses / Competing interests:}

Los autores declaran que no incurre en conflictos de intereses.

\section{Rol de los autores / Authors Roles:}

Edwin Sánchez: conceptualización, curación de datos, análisis formal, adquisición de fondos, investigación, metodología, administración del proyecto, recursos, software, supervisión, validación, visualización, escritura preparación del borrador original, escritura - revisar \& amp; edición.

Oscar Dávila: conceptualización, análisis formal, investigación, metodología, administración del proyecto, recursos, software, supervisión, validación, visualización, escritura - preparación del borrador original, escritura - revisar \& amp; edición.

\section{Fuentes de financiamiento / Funding:}

Los autores declaran que no recibió un fondo específico para esta investigación.

\section{Aspectos éticos / legales; Ethics / legals:}

Los autores declaran no haber incurrido en aspectos antiéticos, ni haber omitido aspectos legales en la realización de la investigación.

\section{REFERENCIAS}

Alonso, L., Hernández, R., Velazco, Y., \& Suárez, L. (2021). La familia en la educación a distancia de los escolares durante la Covid-19. Revista Iberoamericana de Investigación en educación, 2(2), 45-55. https://riied.org/index.php/v1/article/view/19

Arévalo, P., Cruz, J., Guevara, C., Palacio, A., Bonilla, S., Estrella, A., Guadalupe, J., Zapata, M., Jadán, J., Arias, H., \& Ramos, C. (2020). Actualización en metodología de la investigación científica [Universidad Tecnológica Indoaméricca]. http://201.159.222.95/handle/123456789/1686 
Arias, F. (2012). El proyecto de investigación. introducción a la metodología científica. Episteme.

Balsells, Á., Urrea, A., Ponce, C., Vaquero, E., \& Navajas, A. (2018). Claves de acción socioeducativa para promover la participación de las familias en procesos de acogimiento. Educación XX1, 22(1). https://doi.org/10.5944/educxx1.21501

Bellido, J. (2021). Participación de las familias, acción tutorial y orientación desde la justicia social. REOP Revista Española de Orientación y Psicopedagogía, 32(1), 76-91. https://doi.org/10.5944/reop.vol.32.num.1.2021.30741

Bisquerra, R. (2004). Metodología de la investigación educativa. La Muralla.

Bravo, B., \& Oviedo, A. (2020). Estrés emocional debido al aislamiento social por la pandemia covid-19 en padres de familias vulnerables. Revista Científico-Académica Multidisciplinaria, 5(9), 579-591. https://doi.org/10.23857/pc.v5i9.1712

Buitrago, R., \& Molina, G. (2021). Profesorado, emociones y escuela. Reflexiones en tiempo de pandemia covid-19-. Revista Habitus: Semilleros de investigación, 1(1), e12551. https://doi.org/10.19053/22158391.12551

Caballero, L. (2020). Inteligencia emocional: Una guía paso a paso para mejorar su coeficiente emocional, controlar sus emociones y comprender sus relaciones. Crecimiento de Autoayuda.

Castillo, R., Salazar, H., Rodríguez, F., \& Ruán, C. (2021). La educación en tiempos de pandemia: emociones y percepciones en estudiantes de nivel medio superior y licenciatura. Política y Cultura, 51, 121-148. https://polcul.xoc.uam.mx/index.php/polcul/article/view/1452/1408

Colomo, E., Gabarda, V., Cívico, A., \& Cuevas, N. (2021). Ansiedad ante la muerte y felicidad en el alumnado del grado de educación primaria de la Universidad de Málaga. Multidisciplinary Journal of Educational Research, 11(1), 49-76. https://doi.org/10.4471/remie.2021.5865

Corchuelo, C., Cejudo, C., \& Tirado, R. (2019). Las conexiones entre apoyo familiar, escolar y el compromiso de los estudiantes en un centro de compensación educativa//The connections between family support, school and the engagement of th students in a compensatory education school. REOP - Revista Española de Orientación y Psicopedagogía, 30(2), 46. https://doi.org/10.5944/reop.vol.30.num.2.2019.25337

Cruz, P., \& Borjas, M. (2019). Importancia de los primeros pasos en la relación familia-colegio. (Diseño del 
periodo de adaptación en educación infantil). Enseñanza \& Teaching: Revista Interuniversitaria de Didáctica, 37(2), 27. https://doi.org/10.14201/et20193722744

Expósito, C., \& Marsollier, R. (2020). Virtualidad y educación en tiempos de COVID-19. Un estudio empírico en Argentina. Educación y Humanismo, 22(39). https://doi.org/10.17081/eduhum.22.39.4214

Fernández, A. (2020). 2020: Estudiantes, emociones, salud mental y pandemia. Revista Andina de Educación, 4(1), 23-29. https://doi.org/10.32719/26312816.2021.4.1.3

Ferraces, M., Lorenzo, M., Godás, A., \& Santos, M. (2020). Students' Mediator Variables in the Relationship between Family Involvement and Academic Performance: Effects of the Styles of Involvement. Psicología Educativa, 27(1), 85-92. https://doi.org/10.5093/psed2020a19

Flórez, G., Villalobos, J., \& Londoño, D. (2017). El acompañamiento familiar en el proceso de formación escolar para la realidad colombiana: de la responsabilidad a la necesidad. Psicoespacios, 11(18), 196. https://doi.org/10.25057/21452776.888

García, Á. (2021). La enseñanza Online post pandemia: Nuevos retos retos. Holos, 2. https://doi.org/10.15628/holos.2021.12082

Goleman, D. (2002). Inteligencia emocional. Kairós.

Gutiérrez, D., \& Espinel, N. (2021). Educación en pandemia: variables de reflexión, retos y oportunidades. Revista Educación y Ciudad, 41, 119-131. https://doi.org/10.36737/01230425.n41.2579

Guzmán, K., Bastidas, B., \& Mendoza, M. (2019). Estudio del rol de los padres de familia en la vida emocional de los hijos. Apuntes Universitarios, 9(2). https://doi.org/10.17162/au.v9i2.360

Halpern, D., Piña, M., \& Ortega, C. (2021). Mediación parental y escolar: uso de tecnologías para potenciar el rendimiento escolar. Educación XX1, 24(2). https://doi.org/10.5944/educxx1.28716

Hernández, C., Cárdenas, C., Romero, P., \& Hernández, M. (2017). Los padres de familia y el logro académico de los adolescentes de una secundaria en Milpa Alta, Ciudad de México. Información tecnológica, 28(3), 119-128. https://doi.org/10.4067/S0718-07642017000300013

Hernández, R., \& Mendoza, C. (2018). Metodología de la investigación. Las rutas cuantitativa, cualitativa y mixta. McGraw-Hill. 
Lagos, M., \& Luque, M. (2020). Apoyo y participación educativa de las familias de los países del sur de Europa: una aproximación a la realidad española y portuguesa. Revista Española de Sociología, 29(1), 101-115. https://doi.org/10.22325/fes/res.2020.07

Lastre, K., López, L. D., \& Alcazar, C. (2017). Relación entre apoyo familiar y el rendimiento académico en estudiantes colombianos de educación primaria. Psicogente, 21(39). https://doi.org/10.17081/psico.21.39.2825

López, V., Álvarez, J., Calisto, A., Aguilar, G., Barrios, P., Cárdenas, M., Briceño, D., Vera, M., Meneses, H., Romero, B., \& Leiva, M. (2021). Apoyo al bienestar socioemocional en contexto de pandemia por COVID19: sistematización de una experiencia basada en el enfoque de Escuela. F@ro: revista teórica del Departamento de Ciencias de la Comunicación, 1(33). http://www.revistafaro.cl/index.php/Faro/article/view/645

Madueño, P., Lévano, J., \& Salazar, A. (2020). Conductas parentales y habilidades sociales en estudiantes de educación secundaria del Callao. Propósitos y Representaciones, 8(1). https://doi.org/10.20511/pyr2020.v8n1.234

Motha, K. (2018). Educational Support for Orphaned Children: What Can We Learn from the African Extended Family Structure? Children \& Society, 32(1), 50-60. https://doi.org/10.1111/chso.12232

Muñoz, O., \& Lluch, L. (2020). Educación y Covid-19: colaboración de las familias y tareas escolares. Revista Internacional De Educación Para La Justicia Social, 9(3). https://revistas.uam.es/riejs/article/view/12182\#

Ñaupas, H., Valdivia, M., Palacios, J., \& Romero, H. (2013). Metodología de la investigación cuantitativacualitativa y redacción de la tesis (5a.Edición). Ediciones de la U. https://corladancash.com/wpcontent/uploads/2020/01/Metodologia-de-la-inv-cuanti-y-cuali-Humberto-Naupas-Paitan.pdf

Noguera, F. (2020). Padres balanceados, hijos felices. Guía para una cranza amorosa (1. . ed.). Diana.

Pachay, M., \& Rodríguez, M. (2021). La deserción escolar: Una perspectiva compleja en tiempos de pandemia. Polo del Conocimiento, 6(1), 130-155. https://doi.org/10.23857/pc.v6i1.2129

Paris, J. (2020). Emociones, marketink y comunicación: desde el paradigma esencial. Diseño.

Pérez, Y., Barroso, C., Amaro, A., \& Lorenzo, A. (2021). Acciones educativas de la familia con el escolar durante el confinamiento por la COVID-19. Revista Cubana de Pediatría, 93(3). 
http://www.revpediatria.sld.cu/index.php/ped/article/view/1377

Pineda, A., \& Chiappe, A. (2018). ICT and home-educating families: a qualitative multiple case study. Ensaio: Avaliação e Políticas Públicas em Educação, 26(101), 1324-1346. https://doi.org/10.1590/s010440362018002601507

Razeto, A. (2016). El involucramiento de las familias en la educación de los niños. Cuatro reflexiones para fortalecer la relación entre familias y escuelas. Páginas de Educación, 9(2), 190. https://doi.org/10.22235/pe.v9i2.1298

Rodríguez, J. (2013). Paradigmas, enfoques y métodos en la investigación educativa. Revistas de investigación UNMSM, 7(12). https://revistasinvestigacion.unmsm.edu.pe/index.php/educa/article/view/8177

Rojas, P. (2019). Inteligencia y gestión emocional del profesorado en la escuela. Wanceulen S.L.

Rubio, T., \& Garduño, J. (2020). La pandemia y la utopía: hacia nuevos paradigmas educativos. El Cotidiano, 35(221), 17-28. https://www.proquest.com/docview/2462681312

Rueda, N. (2020). La responsabilidad civil en el ejercicio de la parentalidad. Bogotá : Universidad Externado de Colombia. https://bdigital.uexternado.edu.co/handle/001/2782

Sánchez, A., Reyes, F., \& Villarroel, V. (2016). Participación y expectativas de los padres sobre la educación de sus hijos en una escuela pública. Estudios pedagógicos (Valdivia), 42(3), 347-367. https://doi.org/10.4067/S0718-07052016000400019

Sánchez, C., Daura, F., \& Laudadío, J. (2019). La comunidad escolar como medio para el desarrollo socio emocional de los alumnos. Un estudio de caso en la República Argentina. Estudios pedagógicos (Valdivia), 45(3), 31-49. https://doi.org/10.4067/S0718-07052019000300031

Sanz, P. (2020). Cómo enseñar a nuestros hijos a digerir sus emociones. El País. https://elpais.com/elpais/2020/10/20/laboratorio_de_felicidad/1603177657_444833.html

Solís, F., \& Aguiar, R. (2017). Análisis del papel del involucramiento de la familia en la escuela secundaria y su repercusiòn en el rendimiento acadèmico. Sinéctica, 49. http://www.scielo.org.mx/scielo.php?script=sci_arttext\&pid=\$1665-109X2017000200013

Tornés, B. (2021). La educación en valores desde la familia en el contexto actual. Revista Medica de Santiago 
de Cuba, 25(4). http://www.medisan.sld.cu/index.php/san/article/view/3853/2437

Tua, A. (2021). Corresponsabilidad de los padres en el proceso educativo de sus hijos durante confinamiento por covid-19. Revista EDUCARE - UPEL-IPB - Segunda Nueva Etapa 2.0, 25(2), 256-278. https://doi.org/10.46498/reduipb.v25i2.1469

Vaccotti, R. (2019). La relación familia-institución educativa en enseñanza media: perspectivas de docentes de secundaria. Páginas de Educación, 12(1), 164. https://doi.org/10.22235/pe.v12i1.1787

Valdés, M. (2021). El estallido social y sus implicaciones para la situación tributaria en Colombia. https://doi.org/10.33960/AC_23.2021

Vera, A., Cerda, G., Aragón, E., \& Pérez, C. (2021). Rendimiento académico y su relación con variables socioemocionales en estudiantes chilenos de contextos vulnerables. Educación XX1, 24(2). https://doi.org/10.5944/educxx1.28269

Villanueva, H., Vega, P., Vasquez, C., Morales, S., \& Siccha, R. (2021). Percepción del desempeño docente según los actores educativos, en tiempo de pandemia. Espacios, 42(17), 50-60. https://doi.org/10.48082/espacios-a21v42n17p04

Viso, J. (2020). Escuelas que emocionan. Biblioteca innova educación. 